\title{
Many-body localization from a one-particle perspective in the disordered 1D Bose-Hubbard model
}

\author{
Miroslav Hopjan ${ }^{1}$ and Fabian Heidrich-Meisner ${ }^{1}$ \\ ${ }^{1}$ Institute für Theoretische Physik, Friedrich-Hund-Platz 1, 37077 Göttingen, Germany
}

\begin{abstract}
We numerically investigate 1D Bose-Hubbard chains with onsite disorder by means of exact diagonalization. A primary focus of our work is on characterizing Fock-space localization in this model from the single-particle perspective. For this purpose, we compute the one-particle density matrix (OPDM) in many-body eigenstates. We show that the natural orbitals (the eigenstates of the OPDM) are extended in the ergodic phase and real-space localized when one enters into the MBL phase. Furthermore, the distributions of occupations of the natural orbitals can be used as measures of Fock-space localization in the respective basis. Consistent with previous studies, we observe signatures of a transition from the ergodic to the many-body localized (MBL) regime when increasing the disorder strength. We further demonstrate that Fock-space localization, albeit weaker, is also evidently present in the distribution of the physical densities in the MBL regime, both for softand hardcore bosons. Moreover, the full distribution of the densities of the physical particles provides a one-particle measure for the detection of the ergodic-MBL transition which could be directly accessed in experiments with ultra-cold gases.
\end{abstract}

\section{INTRODUCTION}

Closed quantum systems with an interplay of interactions and disorder represent a paradigmatic case of systems where thermalization is believed to fail [1-5]. The original concept of disorder-driven Anderson localization [6] and its generalization to systems of interacting electrons developed into the more generic framework of many-body localization (MBL) $[7,8]$ for closed quantum systems. The delocalization-to-MBL (or ergodic-MBL) transition is an unconventional phase transition at finite energy density, i.e., not related to symmetry and not seen in thermodynamics. It is often referred to as an eigenstate transition [2]. The MBL phase is a state of matter with emergent local integrals of motion [9-12] where eigenstates exhibit area-law entanglement [13-15] and where slow logarithmic entanglement entropy growth can be observed in global quenches $[9,16,17]$. For an overview of this rapidly evolving field, we refer to recent reviews $[1-5]$.

Insights from numerical investigations of MBL in spin$1 / 2$ XXZ chains (or the equivalent model of spinless fermions) $[4,14-26]$ by means of exact diagonalization or by means of tensor-network methods greatly contributed to the current understanding of the MBL phase. Most of the numerical simulations investigated either the properties of the eigenspectrum and the eigenstates and the violation of the eigenstate thermalization hypothesis (ETH), e.g., the level statistics, the number variance, the entanglement entropy, and Fock-space localization, or the realtime evolution after a global quench starting from pure, spatially inhomogeneous initial states. We note that recently, a controversial discussion emerged on whether the existence of MBL can be inferred from finite-size data at all [27-30], also questioning the existence of the MBL phase in the thermodynamic limit [27]. This is related to the intensely discussed question of the exact nature of the transition (see $[26,31-36]$ ). These discussions are ongoing, without a final conclusion yet.

Experimental progress has been made with ultracold atoms [37], trapped ions [38], and superconducting qubits $[39,40]$ where various lattice models with disorder can be emulated. The observation of signatures of the MBL phase was achieved in the quasiperiodic AubryAndré Fermi-Hubbard model [37, 41], the disordered Ising model [38], the disordered Bose-Hubbard model (BHM) $[42,43]$ and the quasiperiodic Aubry-André BoseHubbard model [44, 45]. Recently, the disordered BHM was also realized with interacting photons in an array of superconducting qubits [46]. Most of the experiments carried out with different platforms measure the dynamics of the imbalance decay [37, 41, 42] or the dynamics of the entanglement entropy [44, 46].

However, so far, only a few numerical studies considered the experimentally relevant disordered BHM [47-52] (or the BHM with random interactions [53]). One reason, perhaps, for the lack of numerical studies are the numerical costs: Full exact diagonalization is feasible only for small system sizes and the studies are thus limited to $1 \mathrm{D}$ [47, 48, 50, 52]. For larger 1D or 2D systems, using approximative methods is unavoidable $[49,51]$. Nevertheless, these numerical studies suggest that an MBL phase exists in the disordered 1D BHM. The MBL phase was characterized by, for example, the imbalance decay [47], the entanglement-entropy growth [50], the level statistics of many-body eigenspectra [47, 48, 50], the gap ratio and the fractal dimension statistics of the full low-energy quasiparticle spectra [51], or by the entanglement entropy $[49,50]$. Furthermore, the existence of one (inverted) $[47,49,52]$ or more many-body mobility edges [51] was proposed. Several studies suggest that the existence of double and higher local occupancies may favor localization [54-56], even in the absence of disorder $[57,58]$. The understanding of MBL in the disordered BHM is, however, still far from complete.

Motivated by all these considerations, we here follow an approach based on the one-particle density matrix 
(OPDM) computed in many-body eigenstates [23]. By diagonalising the OPDM, one obtains the natural orbitals and their occupations which can be used to characterize the real-space localization and Fock-space localization, respectively. This has previously been introduced for spinless fermions in [23] and has been studied in [59-64]. As a main result of this analysis, a steplike discontinuity in the disorder-averaged occupations of the natural orbitals was observed, a consequence of Fock-space localization $[8,21,65,66]$. The ergodic phase, by contrast, exhibits a smooth OPDM occupation function, consistent with thermal behavior $[23,59]$.

Here, we extend these ideas to the bosonic case. In particular, we aim at elucidating the connection between Fock-space and real-space localization in the BHM from the one-particle perspective. We first revisit the spin$1 / 2$ Heisenberg model, which is equivalent to a model of hardcore bosons, and which is, at the same time, a standard model for the study of MBL. We demonstrate that by diagonalization of the spin-correlation matrix instead of the fermionic OPDM, we also obtain natural orbitals and a set of eigenvalues, the occupations. The development of a steplike discontinuity in the disorderedaveraged spin-projections and the disordered-averaged occupations of the natural orbitals is observed, analogously to the fermionic case [23] (see also [67]). Furthermore, we define a quantitative Fock-space localization measure from the full distributions of the physical spin-projections and the occupations of natural orbitals. This measure, which we dub occupation distance, quantifies the discrete character of the distributions in the MBL phase, related to the proximity of manybody eigenstates to Slater determinants (permanents) for fermions (bosons). The system-size dependence of this measure is different in the ergodic and the MBL phase and the change in the finite-size dependence occurs close to the transition point estimated from other measures $[21,31,36]$.

In the second part, we focus our investigation on the disordered BHM concentrating on densities relevant for recent experiments [42]. We first consider the entanglement entropy to show that the disordered BHM indeed exhibits the ergodic-MBL crossover consistent with previous studies $[47,48,50]$. Then, we diagonalise the bosonic OPDM to obtain the natural orbitals and their occupations to characterize the real-space localization and Fockspace localization. First, we observe that the natural orbitals are extended in the ergodic phase and real-space localized when one enters into the MBL phase. We show that the disorder-averaged occupations of the natural orbitals exhibit a step-like structure. Furthermore, using our quantitative measure for the degree of Fock-space localization, the occupation distance, we extract information about the Fock-space localization. Analogously to spins, the system-size dependence of the occupation distance is different in the ergodic and in the MBL phase. Interestingly, the Fock-space localization is also evident in the distributions of physical densities, which we anal- yse in the same way as the distributions of the naturalorbital occupations. We argue that this type of analysis of the distribution of physical densities may provide an additional means to investigate MBL and the ergodicMBL transition in quantum-gas experiments.

The plan of the paper is the following. We start with the introduction of the one-particle measures both for the spin- $1 / 2$ case and for bosons in Sec. II. We apply the oneparticle characterization to the 1D spin- $1 / 2$ Heisenberg model in the random magnetic field in Sec. III. Then, we apply the one-particle characterization to the disordered BHM in Sec. IV. We conclude our study in Sec. V.

\section{MODEL AND METHODS}

We first investigate the $1 \mathrm{D}$ spin- $1 / 2$ Heisenberg model with $L$ sites

$$
H=\sum_{i=1}^{L}\left[\frac{J}{2}\left(\hat{S}_{i}^{+} \hat{S}_{i+1}^{-}+\text {H.c. }\right)+J \hat{S}_{i}^{z} \hat{S}_{i+1}^{z}+h_{i} \hat{S}_{i}^{z}\right] .
$$

Here, $\hat{S}_{i}^{+}\left(\hat{S}_{i}^{-}\right)$is a raising (lowering) spin- $1 / 2$ operator at site $i, \hat{S}_{i}^{z}$ measures the $z$-component of the spin and $h_{i}$ represents a random local magnetic field drawn from a box distribution of width $2 W$, i.e., $h_{i} \in[-W, W]$. From now on, all energies are expressed in units of the nearestneighbour spin-exchange constant $J$.

Before we introduce the one-particle measure for spins, we review the one-particle characterization for interacting fermions on a tight-binding chain as originally introduced in Ref. [23]. By virtue of a Jordan-Wigner transformation, Eq. (1) can be rewritten as (up to a constant)

$$
\hat{H}=\sum_{i=1}^{L}\left[-\frac{J}{2}\left(\hat{c}_{i}^{\dagger} \hat{c}_{i+1}+\text { H.c. }\right)+J \hat{n}_{i} \hat{n}_{i+1}+h_{i} \hat{n}_{i}\right] .
$$

where $\hat{c}_{i}^{\dagger}\left(\hat{c}_{i}\right)$ is a creation (annihilation) operator for a fermion at site $i$ and $\hat{n}_{i}=\hat{c}_{i}^{\dagger} \hat{c}_{i}$.

For a given many-body state, $\left|\psi_{n}\right\rangle$, we measure the one-particle density matrix

$$
\rho_{i j}=\left\langle\psi_{n}\left|\hat{c}_{i}^{\dagger} \hat{c}_{j}\right| \psi_{n}\right\rangle .
$$

The natural orbitals $\left|\phi_{\alpha}\right\rangle$ are obtained by diagonalization of the OPDM

$$
\rho\left|\phi_{\alpha}\right\rangle=n_{\alpha}\left|\phi_{\alpha}\right\rangle
$$

The eigenvalues $n_{\alpha}$ are interpreted as occupations of the natural orbitals which sum up to the total number of particles $\sum_{\alpha} n_{\alpha}=N$. We can introduce an associated density operator $\hat{n}_{\alpha}=\hat{c}_{\alpha}^{\dagger} \hat{c}_{\alpha}$, where $\hat{c}_{\alpha}^{\dagger}$ creates a fermion in the natural orbital $\left|\phi_{\alpha}\right\rangle$. In the MBL phase, the natural orbitals exhibit real-space localization and the occupation spectrum reveals the distinctive Fock-space structure of the many-body eigenstates [23]. The occupation spectrum has a steplike structure with most eigenvalues 
close to either one or zero and a discontinuity, thus resembling the momentum distribution of a Fermi liquid $[23,59]$.

We now return to the spin representation as used in Eq. (1). First, we introduce the expectation value of the $z$-component of the spin at site $i$ defined as $s_{i}=$ $\left\langle\psi_{n}\left|\hat{S}_{i}^{z}\right| \psi_{n}\right\rangle$ in a many-body eigenstate $\left|\psi_{n}\right\rangle$. We will argue that the expectation values $s_{i}$ can be used as a measure of both real-space and Fock-space localization. We now introduce the spin-correlation matrix

$$
S_{i j}^{ \pm}=\left\langle\psi_{n}\left|\hat{S}_{i}^{+} \hat{S}_{j}^{-}\right| \psi_{n}\right\rangle,
$$

which is the analog of the OPDM for spinless fermions. Note that the spin-correlation matrix does not transform exactly to the OPDM for spinless fermions under the Jordan-Wigner transformation. Compared to the fermionic OPDM, it acquires additional phases from the string operators. However, the spin-correlation matrix still provides similar information as the OPDM in the case of fermions as we show in Sec. III A. The spincorrelation matrix and the $z$-components are connected via $s_{i}=S_{i i}^{ \pm}-\frac{1}{2}$. form

The spin-correlation matrix is brought to its diagonal

$$
S^{ \pm}\left|\phi_{\alpha}\right\rangle=s_{\alpha}\left|\phi_{\alpha}\right\rangle
$$

where $\left|\phi_{\alpha}\right\rangle$ are the associated natural orbitals with $s_{\alpha}$ being the respective eigenvalues, i.e., their occupations. The eigenvalues $s_{\alpha}$ will be used as a measure for Fockspace localization whereas the natural orbitals $\left|\phi_{\alpha}\right\rangle$ will be used as a measure for real-space localization.

We further investigate the 1D Bose-Hubbard model with $L$ sites

$$
H=\sum_{i=1}^{L}\left[-\frac{J}{2}\left(\hat{a}_{i}^{\dagger} \hat{a}_{i+1}+\text { H.c. }\right)+\frac{U}{2} \hat{n}_{i}\left(\hat{n}_{i}-1\right)+\epsilon_{i} \hat{n}_{i}\right],
$$

where $\hat{a}_{i}^{\dagger}\left(\hat{a}_{i}\right)$ is a creation (annihilation) operator for a boson at site $i$ and $\hat{n}_{i}=\hat{a}_{i}^{\dagger} \hat{a}_{i}$ is the density operator at site $i, U>0$ accounts for on-site bosonic repulsion and $\epsilon_{i}$ represents an on-site (diagonal) disorder drawn from a box distribution, i.e., $\epsilon_{i} \in[-W, W]$. Similarly to spins, from now on, all energies are expressed in units of the nearest-neighbour hopping constant $J$. Note that we use a prefactor of $J / 2$ instead of the usual $J$ in front of the hopping term to facilitate the comparison to the hardcore boson version of the spin Hamiltonian Eq. (1).

For a Bose-Hubbard chain in a given many-body state $\left|\psi_{n}\right\rangle$, we measure the set of real-space site occupations $\left\{n_{i}\right\}$ where the occupation of site $i$ is defined as $n_{i}=$ $\left\langle\psi_{n}\left|\hat{n}_{i}\right| \psi_{n}\right\rangle$. Additionally, we construct the one-particle density matrix (OPDM) $\rho_{i j}$ defined as

$$
\rho_{i j}=\left\langle\psi_{n}\left|\hat{a}_{i}^{\dagger} \hat{a}_{j}\right| \psi_{n}\right\rangle
$$

Note that the OPDM and the site occupancies are connected via $\rho_{i i}=n_{i}$. The natural orbitals $\left|\phi_{\alpha}\right\rangle$ and their occupations $n_{\alpha}$ are obtained by diagonalization of the $\operatorname{OPDM}(\alpha=1, \ldots, L)$

$$
\rho\left|\phi_{\alpha}\right\rangle=n_{\alpha}\left|\phi_{\alpha}\right\rangle
$$

Note the connection between the spins defined in Eq. (1) and the bosons, i.e., the spins can be represented as hardcore bosons: $\hat{S}_{i}^{+}=\hat{a}_{i}^{\dagger}, \hat{S}_{i}^{-}=\hat{a}_{i}$ and $\hat{S}_{i}^{z}=\hat{n}_{i}-$ $1 / 2$ [68]. The hardcore bosons fulfil the commutation relations

$$
\left[\hat{a}_{i}^{\dagger}, \hat{a}_{j}\right]=\left[\hat{a}_{i}^{\dagger}, \hat{a}_{j}^{\dagger}\right]=\left[\hat{a}_{i}, \hat{a}_{j}\right]=0 \quad(i \neq j),
$$

for different sites and the anti-commutation relations

$$
\left\{\hat{a}_{i}^{\dagger}, \hat{a}_{i}\right\}=1 \quad\left\{\hat{a}_{i}^{\dagger}, \hat{a}_{i}^{\dagger}\right\}=\left\{\hat{a}_{i}, \hat{a}_{i}\right\}=0
$$

for the same site [69]. Then, the spin-correlation matrix $S_{i j}^{ \pm}$corresponds to the OPDM $\rho_{i j}$ in the bosonic picture, i.e., $S_{i j}^{ \pm} \rightleftarrows \rho_{i j}$ and $s_{i} \rightleftarrows n_{i}-1 / 2$. This also justifies the use of the spin-correlation matrix. Therefore, we refer to this object as an OPDM as well.

Apart from the one-particle measures, we also compute the bipartite entanglement entropy. We split the system into subsystems $\mathrm{A}$ and $\mathrm{B}$, both of size $L / 2$, and we expand the eigenstate $\left|\psi_{n}\right\rangle$ as $\left|\psi_{n}\right\rangle=\sum_{i} \alpha_{i}\left|\varphi_{i}\right\rangle_{\mathrm{A}}\left|\chi_{i}\right\rangle_{\mathrm{B}}$ where the $\alpha_{i}$ are positive Schmidt coefficients of the expansion and $\left\{\left|\varphi_{i}\right\rangle_{\mathrm{A}}\right\}$ and $\left\{\left|\chi_{i}\right\rangle_{\mathrm{B}}\right\}$ are orthonormal basis sets in A and B. The von-Neumann entropy between the two parts is then defined as the Shannon entropy of the square of the Schmidt coefficients

$$
S_{\mathrm{VN}}=-\sum_{i} \alpha_{i}^{2} \ln \alpha_{i}^{2} .
$$

The models introduced above are investigated on systems of finite sizes up to $L=18$ (and $10^{3}$ disorder realizations) for the Heisenberg model and up to $L=14$ (and $10^{3}$ disorder realizations) for the Bose-Hubbard model and periodic boundary conditions are imposed. For spins, the overall magnetization is kept to be zero $S^{z}=\sum_{i}\left\langle\hat{S}_{i}^{z}\right\rangle=0$ and for bosons, we set the filling to $n=N / L=\sum_{i}\left\langle\hat{n}_{i}\right\rangle / L=0.5$.

For the spin- $1 / 2$ system, we define the target energy density via $\epsilon=\frac{2\left(E-E_{\min }\right)}{E_{\max }-E_{\min }}$, where $E$ is the many-body energy of a particular eigenstate and $E_{\max }$ and $E_{\min }$ are the maximum and minimum energy for each disorder realization, respectively. The energy density $\epsilon=1$ corresponds to the middle of the many-body spectrum. Full exact diagonalization can be used for system sizes up to $L=16$ (spins) and $L=12$ (bosons), yet we also use the shift-and-invert method here to reduce the computational effort. For the largest system sizes considered here, $L=18$ (spins) and $L=14$ (bosons), we exclusively use the shift-and-invert method [70] (without massive lowerupper decomposition parallelisation). We take the six eigenstates closest to the target energy $\epsilon$ for each disorder realization. The definition of an energy density for the BHM is more subtle and will be discussed in Sec. IV A. 

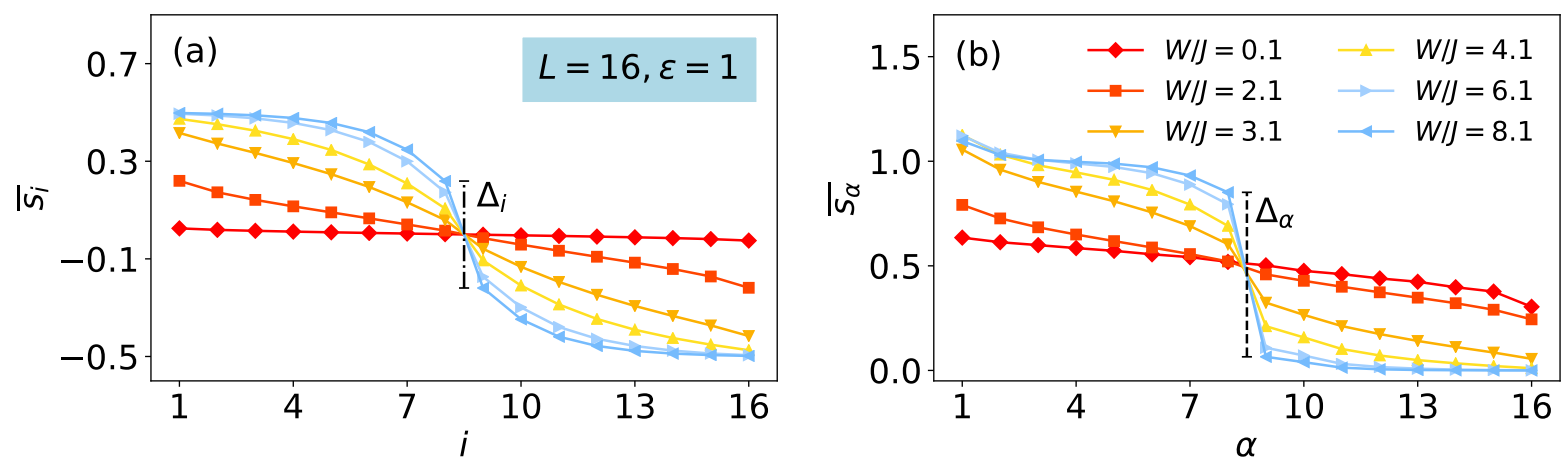

FIG. 1. Spin-1/2 Heisenberg chain: (a) Disorder-averaged spin projection $\overline{s_{i}}$ and (b) disorder-averaged OPDM eigenvalues $\overline{s_{\alpha}}$ for $L=16$ and $\epsilon=1$. Both exhibit gaps $\Delta_{i}$ and $\Delta_{\alpha}$ when first ordered $\left(s_{1} \geq s_{2} \geq \cdots \geq s_{L}\right)$ and then averaged over disorder realizations.

\section{MBL IN THE 1D HEISENBERG MODEL}

\section{A. Disorder-averaged spin projections and OPDM eigenvalues}

We start our discussion with the 1D Heisenberg model. In Fig. 1, we show the values of the disorder-averaged spin projections $s_{i}$ and $s_{\alpha}$, which are first re-ordered from the largest value to the smallest one for each eigenstate. The disorder average is indicated by the bars. We can clearly observe the development of gaps between the values of $\overline{s_{i}}$ and $\overline{s_{\alpha}}$ for $i, \alpha=L / 2$ and $i, \alpha=L / 2+1$ as the disorder strength $W / J$ increases. These gaps are defined as $\Delta_{i}=\overline{s_{i=L / 2}}-\overline{s_{i=L / 2+1}}$ and $\Delta_{\alpha}=\overline{s_{\alpha=L / 2}}-\overline{s_{\alpha=L / 2+1}}$. Such gaps (or occupation discontinuities) were previously reported for spinless fermions [23, 59] and for $S=1 / 2$ spins (and equivalently, for hardcore bosons) [67]. The gaps reflect the fact that the sites and natural orbitals are either nearly occupied or nearly empty, i.e., the particles are more real-space localized and the eigenstates are more Fock-space localized. This is a consequence of the existence of emergent local integrals of motion $[59,67]$ in the MBL phase. It was also argued that the naturalorbital occupations give a better global approximation to the quasiparticle occupations (i.e., the occupations of the local integral of motions) than the site-occupations or the occupations of Anderson orbitals [59]. In this respect, the creation operators of natural orbitals are the closest one to the creation operators of quasiparticles (local integrals of motions) globally [59].

In Fig. 2, we show these gaps as a function of disorder strength $W / J$ and energy density $\epsilon$ for a fixed system size. In the ergodic phase, both $\Delta_{\alpha}$ (shown previously in $[23,59,61])$ and $\Delta_{i}$ need to go to zero as $L$ increases, while the occupation discontinuity is expected to persist in the MBL phase, supported by its $L$-dependence as discussed in [23, 59]. Figures 2(a) and (b) also include the numerical results from [21] for the transition line between the ergodic and the MBL phase extracted from a number of measures (see the caption of Fig. 2 for details). According to these data and at energy density $\epsilon=1$, the transition occurs at about $W_{c} / J \approx 3.6$ [21]. This comparison with the behavior of the gaps is rather encouraging. The crossover is more visible for $\Delta_{\alpha}$ as the natural-orbital occupations are the superior single-particle measure for Fock-space localization [59]. $\Delta_{i}$, however, is the experimentally more accessible quantity as it only requires the measurement of spin projections or densities. This motivates our study of distributions of densities for the disordered BHM.

Before moving on, we remark that it is well-known that (a) $\Delta_{i}$

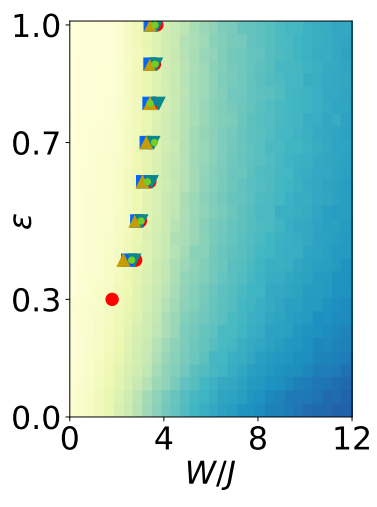

$-S_{V}$ (b) $\Delta_{\alpha}$

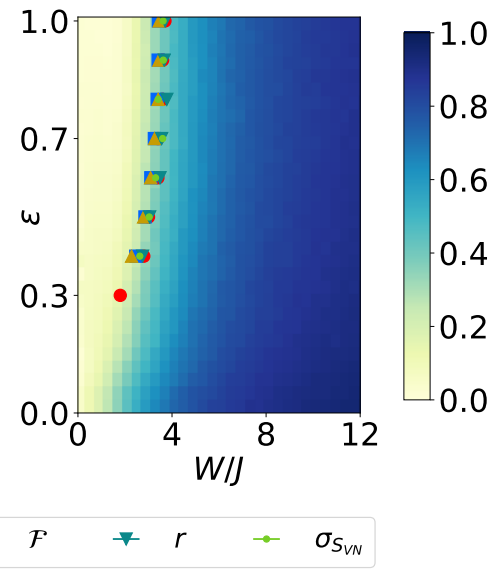

FIG. 2. Spin-1/2 Heisenberg chain: Dependence of the gaps (a) $\Delta_{i}$ and (b) $\Delta_{\alpha}$ on $W / J$ and $\epsilon$ for $L=16$. See Figs. 1(a) and (b) for the definitions of $\Delta_{i}$ and $\Delta_{\alpha}$, respectively. The figures include the data from [21] for the ergodic-to-MBL phase boundary from various measures $\left[S_{V N}\right.$ : an estimate of the boundary between volume and area-law scaling of entanglement entropy, $\mathcal{F}$ : bipartite fluctuations of magnetization, $f$ : the dynamic fraction, $r$ : the ratio of consecutive level spacings, $\sigma_{S_{V N}}$ : entanglement entropy (fluctuations)]. 

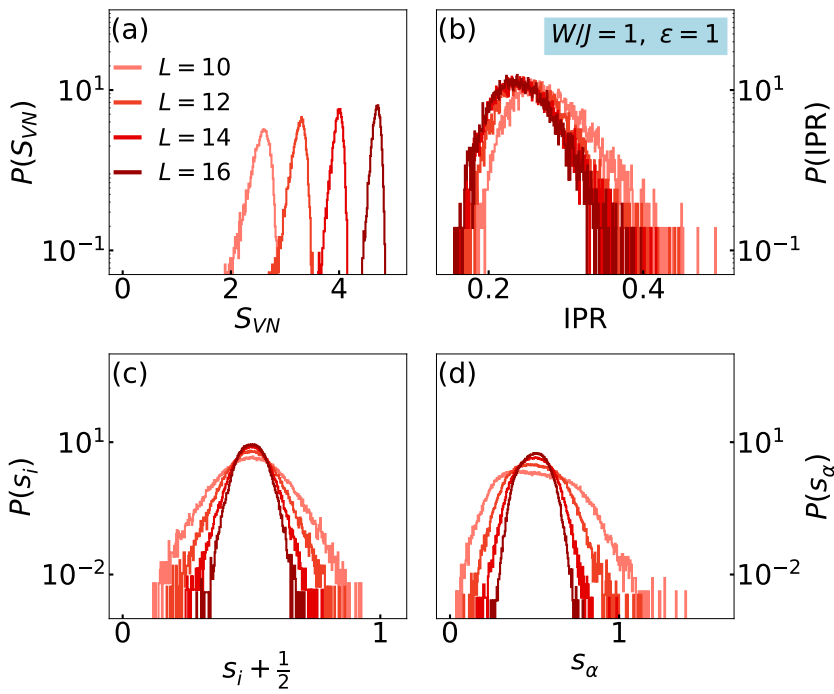

FIG. 3. Spin-1/2 Heisenberg chain: Ergodic phase $(W / J=$ $1, \epsilon=1)$. Full distributions of (a) von-Neumann entanglement entropy, (b) IPR, (c) spin projections and (d) OPDM occupations for $L=10,12,14,16$.

finite-size data extracted from system sizes $L \leq 26$ can suffer from severe finite-size effects in the crossover region [26-30]. Different quantities exhibit different drifts of transition points (see, e.g., [14]). Moreover, there is a range of values reported for the critical disorder strength at, e.g., energy density $\epsilon=1$ in the literature. For instance, numerical linked-cluster expansion simulations [71] or a study of the imbalance decay in Heisenberg chains of $L=100$ spins [72] find substantially larger values for the transition point of $W_{c} / J \approx 4.5$ - 6. More recent studies $[36,73]$ obtain $W_{c} / J \approx 4.2$ with varying error bars. Notably, the results of oneparameter scaling ansatzes (see, e.g., [21]) violate the Harris bound [26, 74, 75], suggesting that the accessible system sizes may not be in the scaling regime yet. Some studies propose estimates of how large system sizes need to be to capture the behavior at the transition (see, e.g., [30]). Even the existence of the MBL phase in the model Eq. (1) is discussed controversially [27-30]. The key issue, though, appears to be that there is no agreement yet on the exact nature of the transition (see, e.g., [26, 31-36] for a discussion).

\section{B. Full distributions of spin projections and OPDM eigenvalues}

To better illustrate the behavior of the one-particle observables, it is instructive to plot the full distributions of $s_{i}$ and $s_{\alpha}$ deep in the ergodic regime [see Figs. 3(c) and (d)] and deep in the localized regime [see Figs. 4(c) and (d)]. At the same time, we also show the distributions of the von-Neumann entanglement entropy $S_{V N}$ in
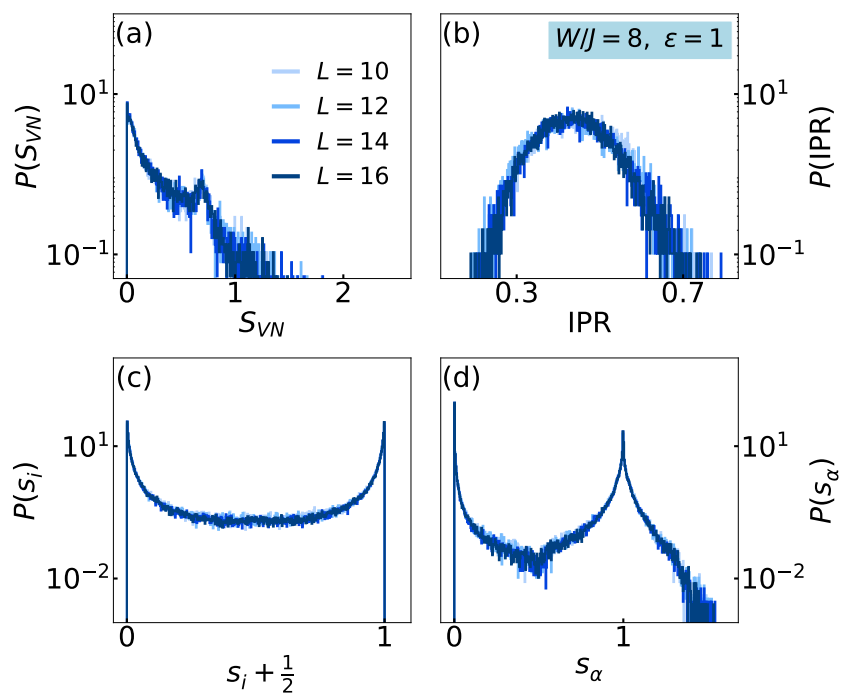

FIG. 4. Spin-1/2 Heisenberg chain: MBL phase $(W / J=$ $8, \epsilon=1)$. Full distributions of (a) von-Neumann entanglement entropy, (b) IPR, (c) spin projections and (d) OPDM occupations for $L=10,12,14,16$.

Figs. 3(a) and 4(a). Finally, we define the inverse participation ratio (IPR)

$$
\mathrm{IPR}=\frac{1}{\left(S^{z}+L / 2\right)} \sum_{\alpha=1}^{L} s_{\alpha} \sum_{i=1}^{L}\left|\phi_{\alpha}(i)\right|^{4}
$$

as a localization measure which contains information about the real-space localization of the natural orbitals $\phi_{\alpha}(i)$. This quantity is shown in Figs. 3(b) and 4(b).

The system-size dependence of the entanglemententropy distributions for spin- $1 / 2$ chains was considered before $[24,76,77]$. On the ergodic side, the maximum of the distribution shifts with system size towards higher values [76] [see Fig. 3(a)]. Close to the transition, long tails of low entanglement entropy develop [76] whereas in the MBL phase, the entanglement entropy distribution does not change with the system size [24] [see Fig. 4(a)]. A similar behavior was found for the $L$-dependence of the IPR. In the ergodic phase, the maximum of the IPR distribution shifts towards lower values [see Fig. 3(b)], while in the localized regime, the IPR distribution does not change with system size [see Fig. 4(b)], consistent with the results for spinless fermions [23].

The distribution of the spin projections $s_{i}$ develops a binary peak structure around the minimal $\left(s_{i}+1 / 2=0\right)$ and maximal $\left(s_{i}+1 / 2=1\right)$ possible values with increasing disorder strength $W / J[24,76,78]$. For low disorder, the distribution depends on system size and becomes sharper as $L$ increases. Moreover, $P\left(s_{i}\right)$ is centered around the average spin projection $\overline{s_{i}}+1 / 2=1 / 2$ [see Fig. 3(c)]. For the larger disorder strength, the distribution is practically $L$-independent [see Fig. 4(c)]. The distribution of the occupations $s_{\alpha}$ show a similar $L$ dependence [see Fig. 3(d) and Fig. 4(d)]. It develops two 


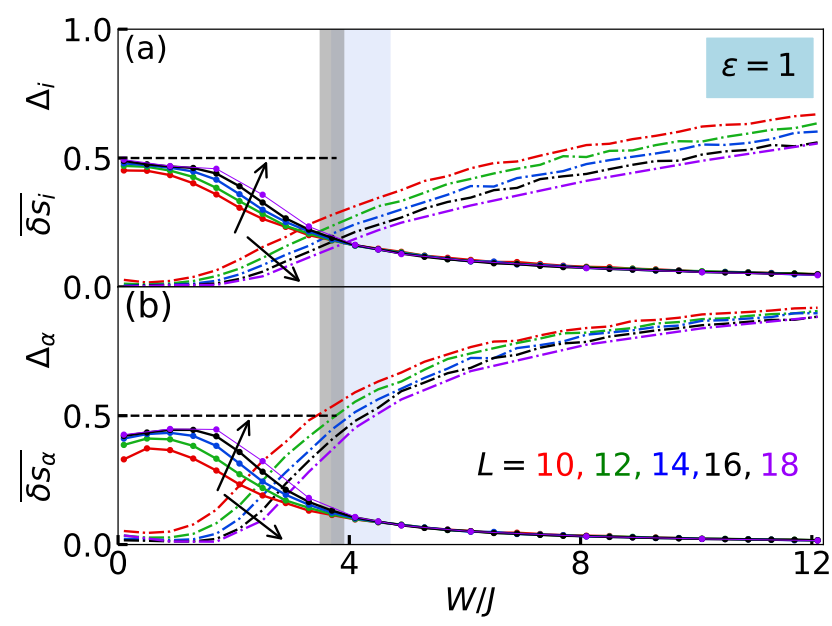

FIG. 5. Spin-1/2 Heisenberg chain: Gaps (a) $\Delta_{i}$ and (b) $\Delta_{\alpha}$ (dashed lines) together with occupation distances, i.e., the average distances (a) $\overline{\delta s_{i}}$ and (b) $\overline{\delta s_{\alpha}}$ (symbols) to the closest integer as a function of $W / J$ for $\epsilon=1$. See Figs. 1(a) and (b) for the definitions of $\Delta_{i}$ and $\Delta_{\alpha}$ and the main text, Sec. III C, for the definitions of $\delta s_{i}$ and $\delta s_{\alpha}$, respectively. The arrows specify increasing system size. The horizontal dashed line in (a) indicates the filling (of hardcore bosons). $\overline{\delta s_{i}}$ is expected to approach this value for $L \rightarrow \infty$ in the ergodic regime. The horizontal dashed line in (b) indicates an upper bound for $\overline{\delta s_{\alpha}}$. For comparison, the vertical lines in grey and blue color mark the position of the ergodic-to-MBL transition estimated from other measures from Refs. [21, 31] and Ref. [36], respectively.

peaks when the disorder strength is increased and the peaks are located around the integer values $s_{\alpha}=\{0,1\}$, reflecting Fock-space localization [23]. We also see that the OPDM occupations can exceed one. This is due to the bosonic character of the spin system, i.e., the spins can be mapped to hardcore bosons and the hardcore bosons do not obey the strict hardcore constraint in the basis of the natural orbitals. Such behaviour was reported before [67].

\section{Quantitative one-particle measure for Fock-space localization}

We have seen that the distributions $P\left(s_{i}\right)$ and $P\left(s_{\alpha}\right)$ develop peak structures around the integers $s_{i}+1 / 2=$ $\{0,1\}$ or $s_{\alpha}=\{0,1\}$, respectively, which reflects Fockspace localization. In order to quantify this aspect, we introduce a measure called occupation distance computed from each element of the distributions. For the OPDM eigenvalues $s_{\alpha}$, this is defined as

$$
\delta s_{\alpha}=\left|s_{\alpha}-\left[s_{\alpha}\right]\right|
$$

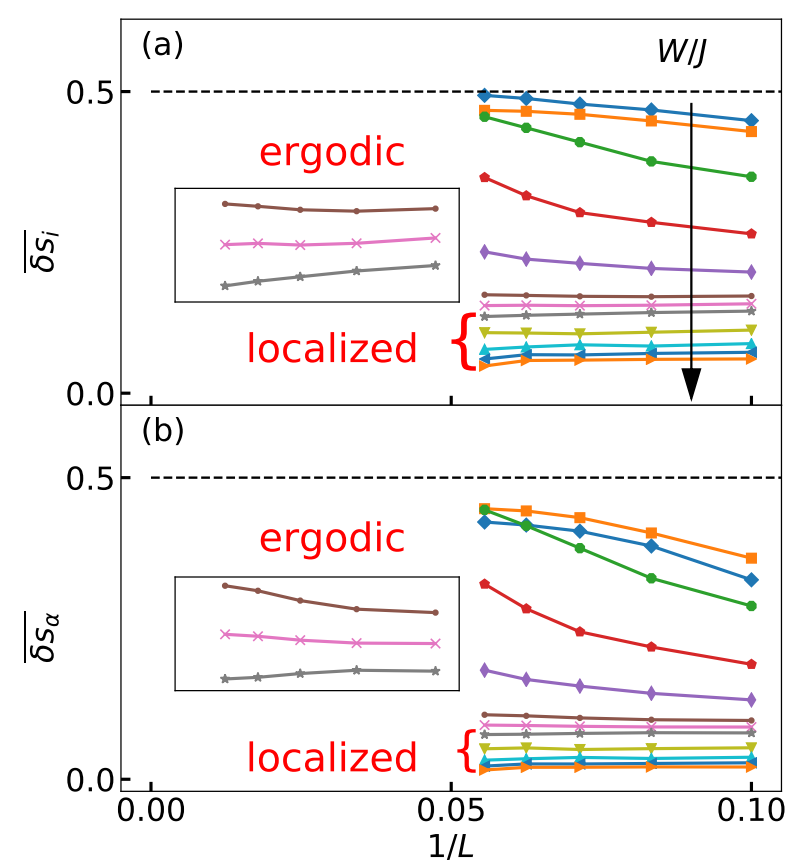

FIG. 6. Spin-1/2 Heisenberg chain: Average occupation distances (a) $\overline{\delta s_{i}}$ and (b) $\overline{\delta s_{\alpha}}$ as a function of $1 / L$ for $\epsilon=1$ for disorder strengths $W / J=0.1,0.9,1.7,2.5,3.3,4.1,4.5,4.9$, $6.1,8.1,10.1,12.1$ (various symbols). The arrow specifies increasing disorder strength. The brackets indicate those data sets that we assign to the localized phase. The insets contain regions zoomed to the data sets for $W / J=4.1,4.5,4.9$ showing the change of the $L$-dependence trends around point $W / J \approx 4.5$.

where $\left[s_{\alpha}\right]$ is the closest integer to $s_{\alpha}$. For the spin projections of physical particles, we alter the definition to

$$
\delta s_{i}=\left|s_{i}+\frac{1}{2}-\left[s_{i}+\frac{1}{2}\right]\right|,
$$

where $\left[s_{i}+1 / 2\right]$ is the closest integer to $s_{i}+1 / 2$. These quantities thus measure the distance to the closest integers, or more generally, the distance to the eigenvalues of the corresponding density operators.

In Fig. 5, we illustrate the dependence of the gaps $\Delta_{i}$ and $\Delta_{\alpha}$ as well as of the disorder-averaged occupation distances $\overline{\delta s_{i}}$ and $\overline{\delta s_{\alpha}}$ on the disorder strength $W / J$ for the energy density $\epsilon=1$. We observe that both gaps $\Delta_{i}$ and $\Delta_{\alpha}$ are increasing functions of the disorder strength and that the gap $\Delta_{\alpha}$ increases faster than the gap $\Delta_{i}$ which reflects the fact that the basis of natural orbitals is the better measure for Fock-space localization. When plotted as a function of $1 / L$ (not shown here), both gaps extrapolate to a finite value for $W \gtrsim 4 J$, with $\Delta_{\alpha}$ extrapolating to larger values than $\Delta_{i}$ (see Ref. [23] for the $L$-dependence of $\Delta_{\alpha}$ ). Moreover, $\Delta_{\alpha}$ goes to zero in the ergodic phase as the OPDM occupation function $n_{\alpha}$ becomes thermal there $[23,59]$. It cannot be ruled out that $\Delta_{i}$ and $\Delta_{\alpha}$ exhibit a discontinuity at the transition. 
The disorder-averaged distances $\overline{\delta s_{i}}$ and $\overline{\delta s_{\alpha}}$ exhibit almost no $L$-dependence for $W / J>4$ while for lower disorder strengths, there is a clear $L$-dependence. To better observe the change of the behavior, we plot the $L$ dependences of $\overline{\delta s_{i}}$ and $\overline{\delta s_{\alpha}}$ as a function of $1 / L$ in Fig. 6 . At weak disorder, $\overline{\delta s_{i}}$ increases with $L$ and approaches 0.5 as $L$ increases, as expected for this magnetization sector $\left(S^{z}=0\right)$. Note that a special case of our $\delta s_{i}$ has recently been studied in [36]. There, specifically $\delta s_{i}^{\min }=$ $1 / 2-\max _{i=1, \ldots, L}\left\{s_{i}\right\}$ has been analyzed, which appears to go to zero as $L$ increases in the MBL phase.

A similar increase with $L$ is observed for $\overline{\delta s_{\alpha}}$, where now 0.5 is an upper bound for $\overline{\delta s_{\alpha}}$. Since the distribution of $s_{\alpha}$ is temperature dependent in the ergodic phase [59], the limit $\overline{\delta s_{\alpha}} \rightarrow 0.5$ is only reached at exactly infinite temperature. Note that the limit of $\delta s_{i}$ and $\delta s_{\alpha}$ that is approached in the ergodic phase depends sensitively on the magnetization sector. We will return to this point in the discussion of the BHM. For strong disorder, $\overline{\delta s_{i}}$ and $\overline{\delta s_{\alpha}}$ seem to saturate to values much smaller than 0.5.

Remarkably, the point separating these two different $L$-dependences of $\overline{\delta s_{i}}$ and $\overline{\delta s_{\alpha}}$ is close to the estimate of the ergodic-MBL transition point extracted from other measures in Ref. [21] or recently from the multifractal scaling theory discussed in Refs. [31, 36]. The data in the insets of Fig. 6 show an increase with $L$ for $W / J=4.1$ but a decrease with $L$ for $W / J=4.9$, while there is no clear $L$-dependence for $W / J=4.5$ suggesting that the change of the behaviour happens somewhere in the interval $W / J \in(4.1,4.9)$. Thus, there is consistency of our data with those other recent finite-size studies [31, 36] even though one cannot exclude a drift of the transition point due to finite size-effects $[26-30,71-73]$. The results presented above suggest that $\overline{\delta s_{i}}$ and $\overline{\delta s_{\alpha}}$ are useful quantitative measures for the degree of Fock-space localization (and better suited than $\Delta_{i}$ and $\Delta_{\alpha}$ ) and motivate us to use analogous measures to study the Fock-space localization in the disordered BHM.

\section{MBL IN THE 1D BOSE-HUBBARD MODEL}

\section{A. Technical aspects and definition of an energy density}

We now turn our discussion to the disordered BHM. Since we consider systems of finite size $L$ with particle numbers $N=L / 2$ and without any hardcore constraint, the local Fock space grows linearly with system size, where $M_{\text {loc. }}=\{0,1,2,3, \ldots, N=L / 2\}$. For $L=$ 8, 10 and 12, we construct the Hamiltonian in the full many-body basis of size $M=330,2002$ and 12376, respectively [79, 80]. For $L=14$, we perform truncations of the local site occupations in the basis states to 2 and 3 bosons (resulting in manageable sizes of the many-body basis of $M=45476$ and $M=69680$, respectively).

In Fig. 7, we show a sketch of the typical eigenspectrum for a system in the low-interaction $(U / J=1)$ regime (a)

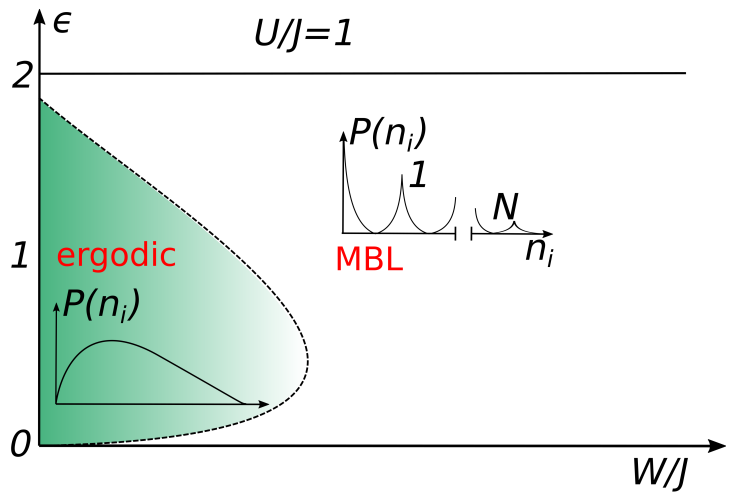

(b)

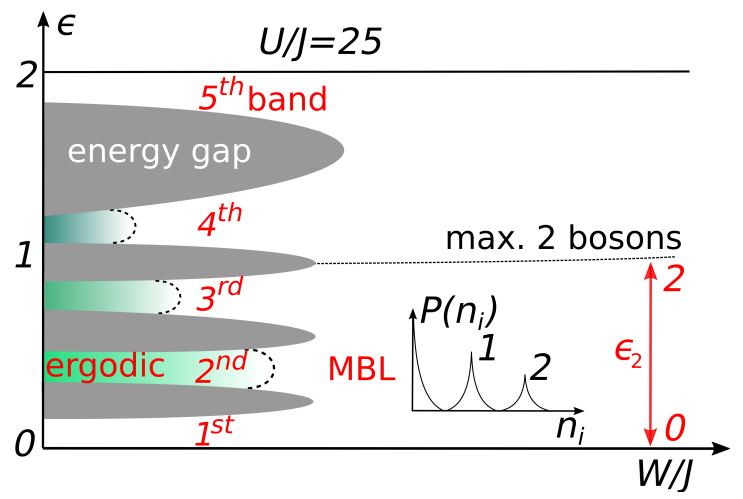

FIG. 7. Bose-Hubbard model: Sketch of the many-body eigenspectra in the $\epsilon-W$ plane for (a) $U / J=1$ and (b) $U / J=25$. The sketch corresponds to chains of size $L=8$ where, for low disorder, the spectra develop five bands in the highinteraction limit $U / J=25$ and where the 3 lowest bands can be characterised by the energy density $\epsilon_{2}$ defined over the sector of eigenstates with maximally 2 bosons per site.

[see Fig. $7(\mathrm{a})]$ and in the high-interaction $(U / J=25)$ regime [see Fig. $7(\mathrm{~b})$ ]. The large-interaction regime is more relevant for the actual experiments [42]. For the low-interaction regime $(U / J=1)$, the spectrum appears to be continuous. On finite systems, in the highinteraction limit $(U / J=25)$, and for low disorder, the spectrum is divided into well separated bands. The bands are determined by the interaction energies of their eigenstates. Typically, the $L$ highest eigenstates in the highest band [see Fig. 7(b)] correspond to configurations with $N$ bosons occupying mostly one site. By going lower in energy in the many-body spectrum, the bosons are allowed to be delocalized. The configurations in the lowest bands [see Fig. 7(b)] can accommodate typically 1 or 2 bosons per site, respectively.

For a system of finite size, the many-body spectrum has a maximum energy, which is a function of the total boson number $N$ and consequently, the BHM with a fixed filling has an unbounded energy per site in the thermodynamic limit. In the highest-energy states, all bosons are located mostly at the same site and energies of such states are approximately given by $E_{\max } \approx U N(N-1) / 2$. Then, considering the filling with $N=L / 2$, the max- 

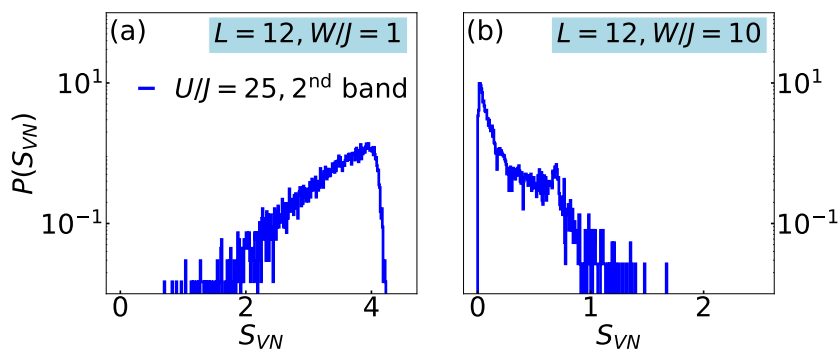

FIG. 8. Bose-Hubbard model: Full distributions of the entanglement entropy of the $2^{\text {nd }}$ band $(L=12, U / J=25, \epsilon=0.15)$ (a) in the ergodic phase and (b) in the MBL phase.

imum energy can be written as $E_{\max } \approx U L(L-2) / 8$ and thus the maximum energy per site of such states $E_{\max } / L \approx U(L-2) / 8$ is a linear function of the system size $L$. This is different from the case of hardcore bosons where the maximum energy per site is bounded from above. One has to keep this in mind when considering the definition $\epsilon=\frac{2\left(E-E_{\min }\right)}{E_{\max }-E_{\min }}$ from Sec. II where now $\epsilon$ cannot be taken as the energy density.

To obtain a quantity which can be interpreted as an energy density, we look at only the part of the spectrum up to a chosen maximal average energy per site. For the system sizes studied here (up to $L=14$ ), we consider states with at maximum doubly-occupied sites as such states (for $L=14$, these are the states which have 7 bosons and 3 doubly-occupied sites). The corresponding energy density $\epsilon_{2}$ is defined as $\epsilon_{2}=\frac{2\left(E-E_{\min }\right)}{E_{\max }^{2}-E_{\min }}$ with respect to the maximum energy of the selected part of the spectrum $E_{\max }^{2}$ [see Fig. 7(b) for an illustration]. In practice, we first compute the size of the truncated basis $M_{\text {red. }}$ by selecting all basis state which have the local occupancy truncated to 2 . We then construct and diagonalize the Hamiltonian in the basis of size $M$ and finally, we compute the energy density $\epsilon_{2}$ with respect to the $M_{\text {red. }}$ lowest eigenenergies.

One should note that with an increasing number of sites the number of bands in the $\epsilon_{2}$ sector of the manybody spectra, as defined above, also increases. In the thermodynamic limit, the number of bands will be infinite and the bands will span the whole range of $\epsilon_{2}$. However, for the system sizes considered here, the bands remain well separated for low disorder. In the following, we focus on the energy density of the second band that roughly corresponds to the middle part of the $\epsilon_{2}$ sector, i.e., $\epsilon_{2} \approx 1$ [see Fig. $7($ b)] and we discuss the numerical signatures of the ergodic-to-MBL transition there.

\section{B. Entanglement entropy}

The first quantity we look at is the bipartite entanglement entropy as a measure for the ergodic-MBL transition [13]. In Fig. 8, we show representative results for
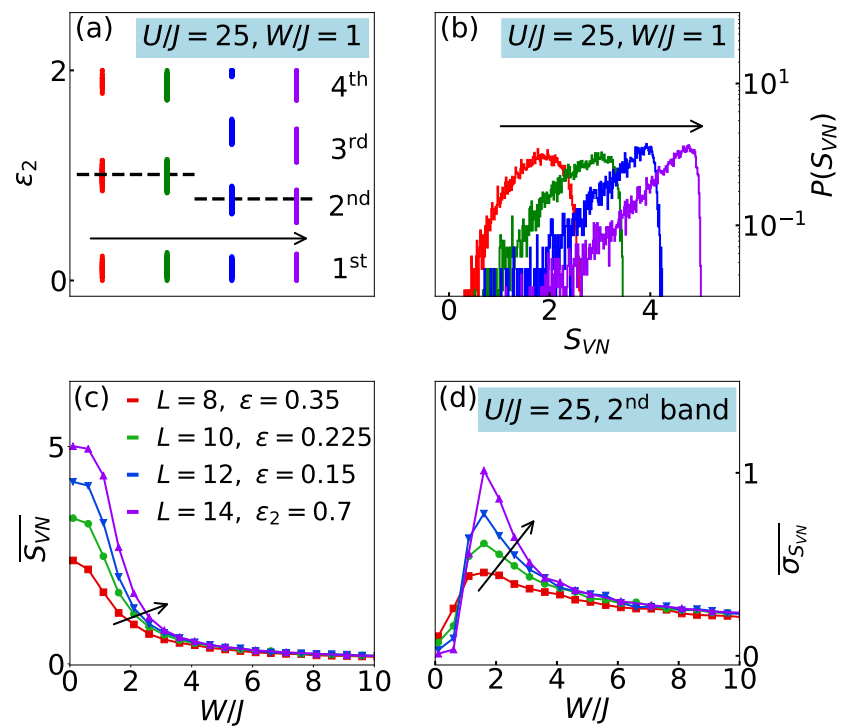

FIG. 9. Bose-Hubbard model: (a) Typical bands of the manybody eigenspectrum expressed in the energy density $\epsilon_{2}$ defined over the sector of eigenstates with maximally 2 bosons per site for system sizes of $L=8,10,12,14$ (with the $L=14$ data from the truncated basis). The arrows specify increasing system size. The dotted lines denote the energy densities of the $2^{\text {nd }}$ bands used for the $L$-dependence analysis in (b), (c) and (d). In (b), we plot the $L$-dependence of the full distributions of the von-Neumann entanglement entropy for the parameters corresponding to the dotted line in (a). In (c), we plot the $L$-dependence of the average entanglement entropy $\overline{S_{V N}}$ as a function of $W / J$. In (d), we plot the $L$-dependence of the average fluctuation $\overline{\sigma_{S_{V N}}}$ of the entanglement entropy as a function of $W / J$. The arrows specify increasing system size.

$L=12$ in the second lowest band for $U / J=25$. For the low disorder $W / J=1$ [see Fig. 8(a)], the entanglemententropy distributions have a maximum at a finite value which is the typical shape of this distribution in an ergodic system [76]. For higher disorder [see Fig. 8(b)], the distribution takes the typical shape in the MBL phase with a maximum close to zero and a local maximum around $S_{V N}=\ln (2)[24,76]$.

The arrows specify increasing system size.

In Fig. 9, we show the $L$-dependence of the entanglement entropy. The second lowest bands for $L=$ $8,10,12$ and 14 have a similar energy density $\epsilon_{2}$ [see Fig. 9(a)]. For low disorder $W / J=1$, the distributions of the entanglement entropy exhibit a shift of the position of their maxima towards higher values [see Fig. 9(b)]. This is the typical $L$-dependence in the ergodic regime [76]. At high disorder $W / J=10$, the distribution is $L$-independent (not shown). In Figs. 9(c) and (d), we plot the average entanglement entropy $\overline{S_{V N}}$ and the average fluctuation $\overline{\sigma_{S_{V N}}}$ of the entanglement entropy as a function of the disorder strength $W / J$, respectively. We observe a large and system-size dependent average entropy for values $W / J \in(0,2.5)$. By contrast, for val- 

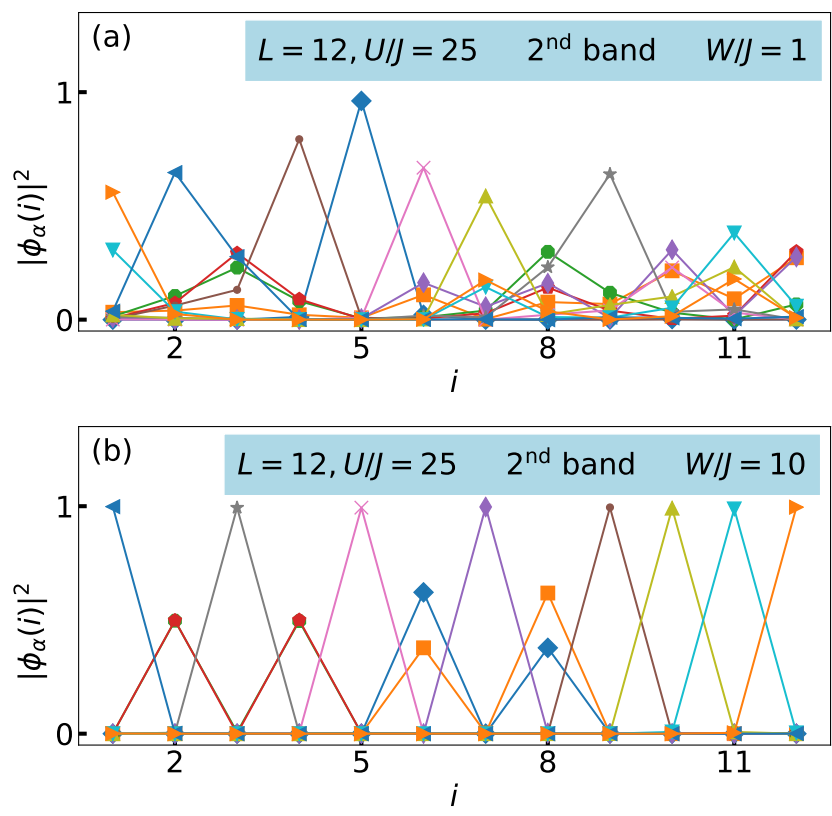

FIG. 10. Bose-Hubbard model: Example of all natural orbitals of the OPDM (various symbols) computed from one randomly chosen eigenstate of the $2^{\text {nd }}$ band $(L=12, U / J=25, \epsilon=$ $0.15)$ (a) in the ergodic $(W / J=1)$ and (b) in the MBL regime $(W / J=10)$.

ues $W / J>4$, the entropy is close to zero for all considered system sizes. This is also reflected in the fluctuation of the entanglement entropy which has a maximum value close to $W / J \approx 2$. This maximum shifts to larger values with increasing system size. The large fluctuations of the entanglement entropy are usually interpreted as a numerical signature of the ergodic-MBL transition and they are expected to diverge at the transition for $L \rightarrow \infty$ [14]. Thus, from the visual inspection of our finite-size numerical data, we can estimate that the transition happens somewhere at $W_{c} / J \approx 2$. By using the one-parameter scaling ansatz of Refs. [21, 26], namely $\overline{S_{V N}} / S_{V N}^{\text {Page }}=g\left[L^{\frac{1}{\nu}}\left(W-W_{c}\right)\right]$, where $S_{V N}^{\text {Page }}$ is the Page value for a random pure state [81], we find an estimate for the transition point of $W_{c} / J=2.0(1)$. However, similarly to the study of spins in Ref. [21], the estimate for the exponent $\nu=0.80(5)$ violates the Harris bound $[26,74,75]$ and one can expect that the true transition point is at a higher value of $W / J$ than the one obtained from the one-parameter scaling estimate.

\section{Natural orbitals and IPR}

In this subsection, we show that the ergodic-MBL transition is also reflected in properties of the natural orbitals. In Fig. 10(a), we plot all natural orbitals for one randomly chosen eigenstate in the ergodic phase for a low disorder strength $(W / J=1)$, while in Fig. 10(b), we
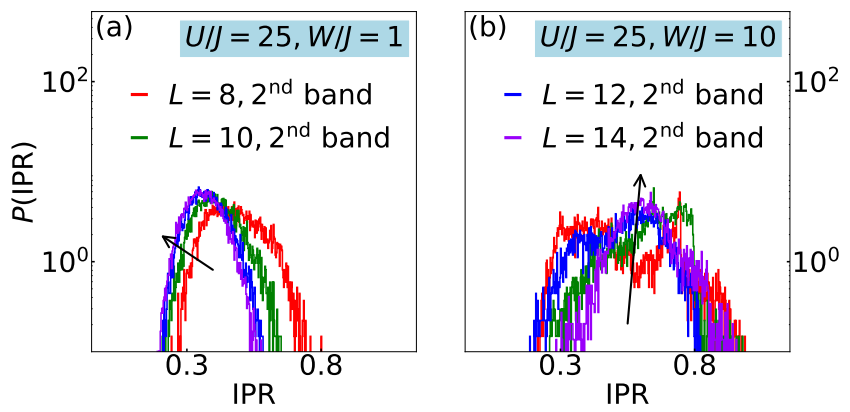

FIG. 11. Bose-Hubbard model: System-size dependence of the full distributions of the IPR (a) in the ergodic phase and (b) in the MBL phase. The parameters correspond to the energy densities denoted by the dotted line in Fig. 9(a), i.e., to the $2^{\text {nd }}$ band of the many-body eigenspectra. The arrows specify increasing system size.

plot all the natural orbitals for one eigenstate in the MBL phase for a high disorder strength $(W / J=10)$. For low disorder, the natural orbitals are delocalized spanning the whole system [see Fig. 10(a)]. On the other hand, from Fig. 10(b), a localization of the natural orbitals by disorder can clearly be observed, similar to the localization of the natural orbitals for fermionic systems.

Following Ref. [23], we define the IPR for bosons as

$$
\mathrm{IPR}=\frac{1}{N} \sum_{\alpha=1}^{L} n_{\alpha} \sum_{i=1}^{L}\left|\phi_{\alpha}(i)\right|^{4} .
$$

The IPR measures the real-space localization of the natural orbitals $\left|\phi_{\alpha}\right\rangle$. In Fig. 11, we show the $L$-dependence of the IPR in the second lowest band (for $U / J=25$ ) for the same parameters as in Fig. 9. For low disorder $W / J=1$, the IPR distribution has a maximum for lower values of IPR with a high-IPR tail which means that the orbitals are mostly delocalized. The distribution of the IPR exhibits a shift in the position of its maximum towards lower values with increasing system size [see Fig. 11(a)]. In the high-disorder regime $W / J=10$, the maxima of the IPR distributions are closer to the maximum value of 1 meaning that the orbitals are mostly localized. Moreover, in the large-disorder regime, the IPR distributions are almost $L$-independent [see Fig. 11(b)]. This is consistent with the behavior of the IPR distributions for fermionic systems [23].

\section{Occupations}

In the previous subsection, we have seen that the natural orbitals contain information about real-space localization. In this subsection, we focus on how the occupations, both of the physical sites $|i\rangle$ and of the natural orbitals $\left|\phi_{\alpha}\right\rangle$, reveal the degree of Fock-space localization.

In analogy to the discussion of the spin model, we first consider the disorder-averaged occupations. In Fig. 12, 

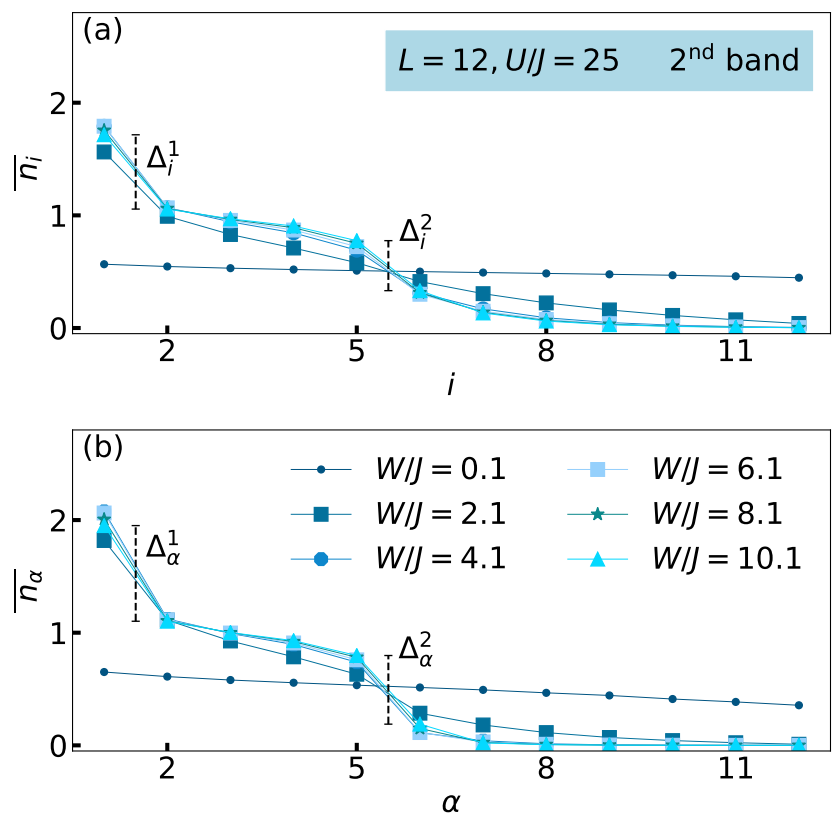

FIG. 12. Bose-Hubbard model: Disorder-averaged and ordered (a) real-space occupations and (b) occupations of natural orbitals for the $2^{\text {nd }}$ band $(L=12, U / J=25, \epsilon=0.15)$. Both exhibit gaps $\Delta_{i}^{j}$ and $\Delta_{\alpha}^{j}(j=1,2)$ when first ordered according to $n_{1} \geq n_{2} \geq n_{3} \geq \cdots \geq n_{L}$ and then averaged over disorder realizations. The vertical dashed lines indicate the location of these discontinuities

we show the disorder-averaged occupations for the second band for $L=12$. For low disorder, the average occupations are a smooth decreasing function. For high disorder, we observe that the averaged occupations exhibit a step-like structure where the occupations are mostly close to 0,1 or 2 . The height of each step between these values is denoted as gaps $\Delta_{i}^{j}$ or $\Delta_{\alpha}^{j}(j=1,2)$ [see Fig. 13]. These gaps are analogous to the gaps observed for spins (or hardcore bosons) and fermions. In the following, we concentrate on the distributions and the occupation distances as they are better-suited measures for Fock-space localization.

Examples of the distributions of the occupations $n_{i}$ and $n_{\alpha}$ for the second band for $L=12$ are displayed in Fig. 13. The first to be noted is that the distributions in the low-disorder regime [see Figs. 13(a) and (b)] are smooth functions with maxima close to the average density of 0.5 and with exponentially decaying tails. In the high-disorder regime, we observe the development of a peak structure. The peaks are located at the integer values $j \in\{0,1,2\}$. Higher occupations in the eigenstates are strongly suppressed which is in agreement with the interaction-energy contribution to the energy of the eigenstates in this particular band. The development of the peak structure in the distributions reflects the ergodic-MBL transition. Thus, analogously to the distributions of $s_{i}$ and $s_{\alpha}$ in the spin system discussed
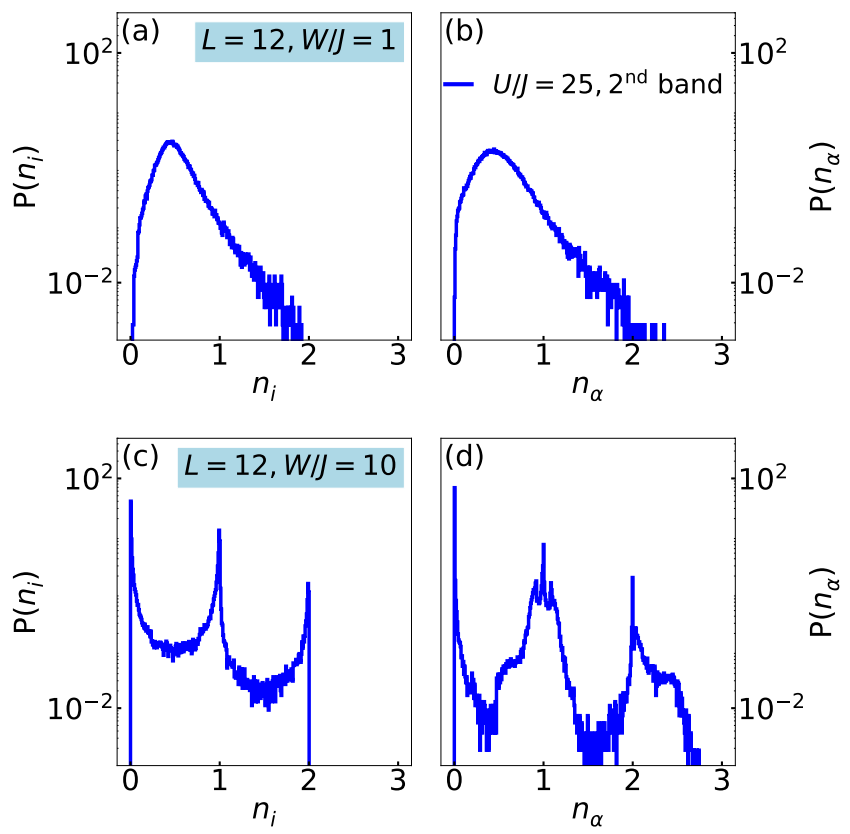

FIG. 13. Bose-Hubbard model: Full distributions of the realspace occupations $n_{i}$ (a) in the ergodic and (c) in the MBL regime and of occupations of natural orbitals $n_{\alpha}(\mathrm{b})$ in the ergodic and (d) in the MBL regime for the $2^{\text {nd }}$ band $(L=$ $12, U / J=25, \epsilon=0.15)$.

above, the distributions of $n_{i}$ and $n_{\alpha}$ indeed reveal the structure of the Fock-space localization. The distribution of $n_{i}$ also indicates real-space localization.

\section{E. Quantitative measure of Fock-space localization}

We have seen that the distributions of the site occupations $n_{i}$ and the natural-orbital occupations $n_{\alpha}$ exhibit a peak structure in the high-disorder regime which reflects many-body localization. To better quantify the localiza-
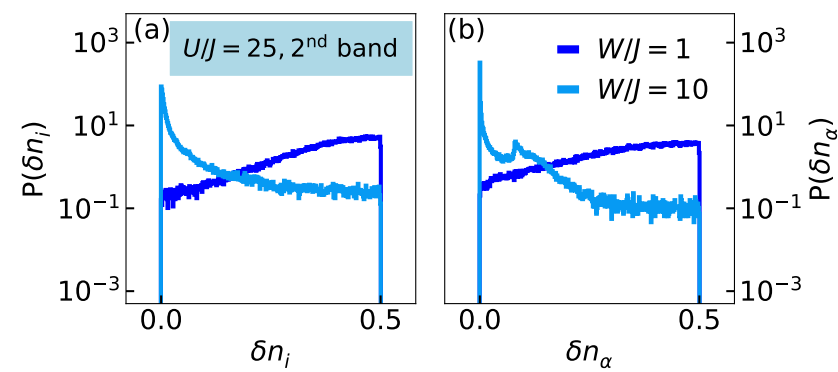

FIG. 14. Bose-Hubbard model: Full distributions of the occupation distances $\delta n_{i}$ of density and $\delta n_{\alpha}$ of natural-orbital occupations in the ergodic $(W / J=1)$ and in the MBL $(W / J=10)$ regimes obtained from the distributions shown in Fig. 13. 


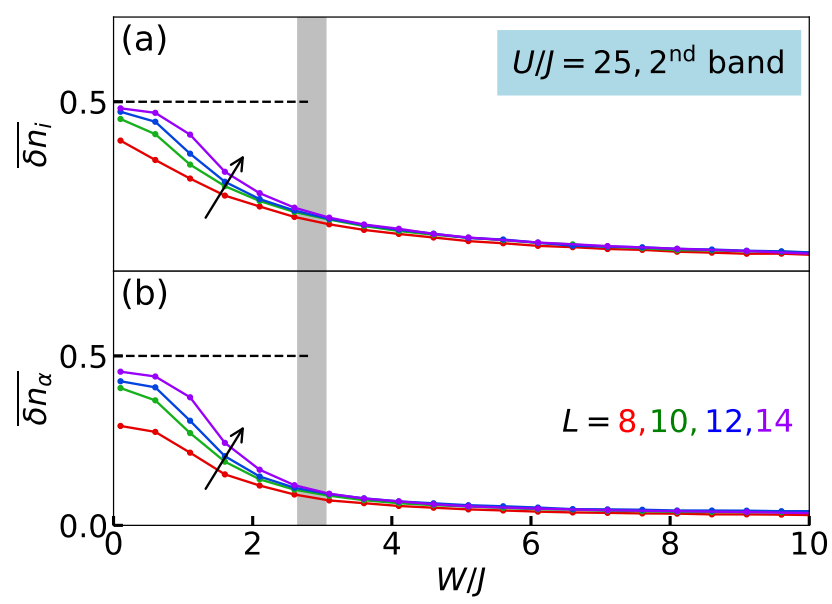

FIG. 15. Bose-Hubbard model: System-size dependence of the average occupation distance (a) $\overline{\delta n_{i}}$ of densities and (b) $\overline{\delta n_{\alpha}}$ of natural-orbital occupations a function of $W / J$. The arrows specify increasing system size. The parameters correspond to the energy densities denoted by the dotted line in Fig. 9(a), i.e., to the $2^{\text {nd }}$ band of the many-body eigenspectra. The horizontal dashed line in (a) indicates the filling. The $\overline{\delta n_{i}}$ is expected to approach this value for $L \rightarrow \infty$ in the ergodic regime. The horizontal dashed line in (b) indicates the upper bound for $\overline{\delta n_{\alpha}}$. The vertical line in grey color marks the estimate of the ergodic-MBL transition estimated from visual inspection of the data in Fig. 16.

tion, we measure, similarly as for the spin system, the distance to the closest integer of the site occupations

$$
\delta n_{i}=\left|n_{i}-\left[n_{i}\right]\right|
$$

and the distance to the closest integer of the occupations of natural orbitals

$$
\delta n_{\alpha}=\left|n_{\alpha}-\left[n_{\alpha}\right]\right|,
$$

where $\left[n_{i}\right]$ and $\left[n_{\alpha}\right]$ are the closest integer to $n_{i}$ and $n_{\alpha}$, respectively. The results for the distributions of $\delta n_{i}$ and $\delta n_{\alpha}$ for the second band for $L=12$ are displayed in Fig. 14 and they show the shift of the maximum of the distribution from 0.5 to 0 with increasing disorder strength. Note that for both quantities, $\delta n_{i}, \delta n_{\alpha} \leq 0.5$.

In Fig. 15, we show $\overline{\delta n_{i}}$ and $\overline{\delta n_{\alpha}}$ as functions of disorder strength $W / J$ for the second band, i.e., for the same parameters as in Fig. 9 and for different system sizes. We observe that the values of $\overline{\delta n_{i}}$ and $\overline{\delta n_{\alpha}}$ are $L$-dependent for the disorder strength $W / J \lesssim 3$ while they are essentially $L$-independent for $W / J \gtrsim 3$.

To better detect the change of the behavior, we illustrate the $L$-dependences of $\overline{\delta n_{i}}$ and $\overline{\delta n_{\alpha}}$ as a function of $1 / L$ in Fig. 16. Clearly, for $W / J \leq 2.6$, the values of $\overline{\delta n_{i}}$ and $\overline{\delta n_{\alpha}}$ are increasing functions of $L$ and $\overline{\delta n_{i}}$ is expected to approach the upper bound $1 / 2$ for $L \rightarrow \infty$, consistent with the data. On the other hand, for $W / J \geq 3.1$ the values of $\overline{\delta n_{i}}$ and $\overline{\delta n_{\alpha}}$ appear to saturate to values much

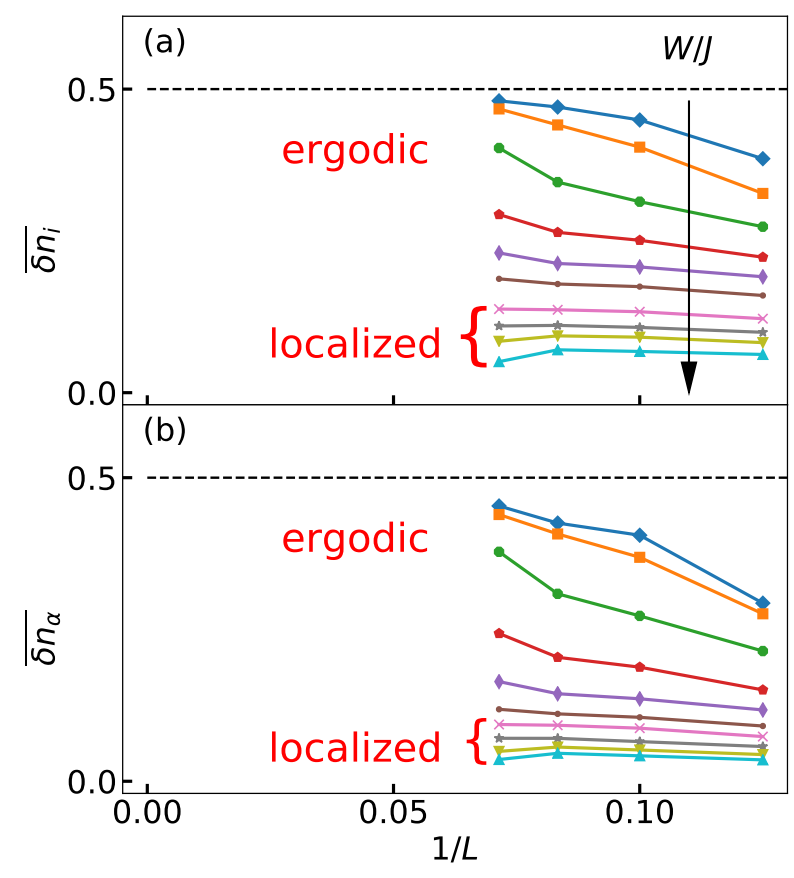

FIG. 16. Bose-Hubbard model: Average occupation distances (a) $\overline{\delta n_{i}}$ and (b) $\overline{\delta n_{\alpha}}$ as a function of $1 / L$ for $\epsilon=1$ for disorder strength $W / J=0.1,0.6,1.1,1.6,2.1,2.6,3.1,4.1,6.1,10.1$ (various symbols). The arrow specifies increasing disorder strength. The brackets indicate those data sets that we assign to the localized phase.

smaller than $1 / 2$ as a function of $L$. From the visual inspection of the data in Fig. 16, the behavior changes for $W / J<3.1$ and we estimate that the transition happens at $2.6<W_{c} / J<3.1$. This is slightly higher than our estimate from the one-parameter scaling of the entanglement entropy of $W_{c} / J \approx 2.0(1)$.

The actual values that $\overline{\delta n_{i}}$ and $\overline{\delta n_{\alpha}}$ approach in the ergodic phase clearly depend on filling. For instance, at unit filling, one expects $\overline{\delta n_{i}} \rightarrow 0$, while $\overline{\delta n_{\alpha}}$ is expected to go to a small but energy-dependent value. One can introduce a modified occupation distance

$$
\tilde{\delta}_{\nu}=\left|n_{\nu}-n\right|
$$

where $\nu=i, \alpha$ and $n$ is the average density or filling. $\tilde{\tilde{\delta}}_{i}$ must approach zero in the ergodic phase but remains finite in the MBL phase. For $\tilde{\delta}_{\alpha}$, we expect a small but in general nonzero value in the ergodic phase and a larger limiting value in the MBL phase compared to $\tilde{\delta}_{i}$. We have verified this behavior for $n=0.5$ yet observe that the finite-size dependencies of $\tilde{\delta}_{\nu}$ are larger than for $\overline{\delta_{\nu}}$.

As a remark, we mention that the regimes where the occupations can reach values larger than 2 can be studied in a similar fashion as the states of the second band. In Fig. 17, we show an example of the distributions for $L=12$ and weak interaction strength in the highdisorder regime $(U / J=1, W / J=10)$ for states from 

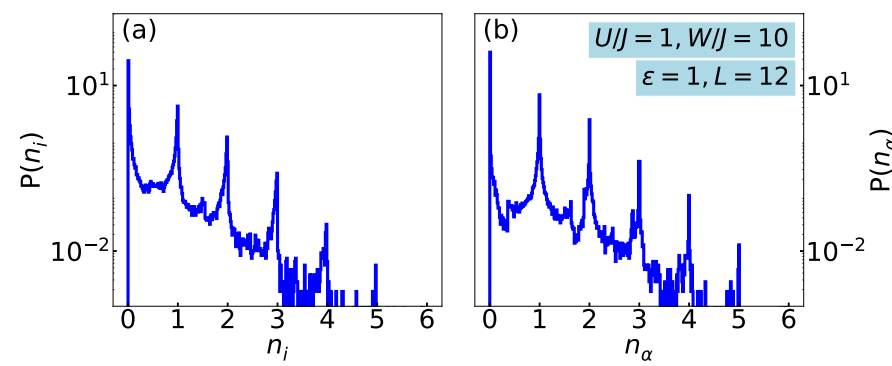

FIG. 17. Bose-Hubbard model: Example for the behavior in the low-interaction regime $U / J=1$. Full distributions of (a) the real-space occupations $n_{i}$, and (b) occupations of natural orbitals $n_{\alpha}$, in the MBL regime for the middle of the manybody eigenspectrum $(L=12, \epsilon=1)$.

the middle of the spectrum. We observe an analogous peak structure in the distributions of $n_{i}$ and $n_{\alpha}$ with the peaks located around integers $j \in\{1,2,3,4,5\}$ (with exponentially decreasing weights of the peaks) showing the localization in Fock space. The relative weight of the height of the peaks depends on energy density, filling, disorder, and interaction strength.

We conclude that the $L$-dependences of the average occupation distances $\overline{\delta n_{i}}$ and $\overline{\delta n_{\alpha}}$ are useful measures for Fock-space localization in the MBL phase. Analyzing the monotony behavior of the $L$-dependence yields a reasonable estimate for the critical disorder strength, consistent with other measures.

\section{F. Measuring densities in quantum-gas experiments}

A measurement of $P\left(n_{i}\right)$ should be feasible with quantum-gas microscopes [37, 41, 42, 44, 45]. In order to obtain the densities $n_{i}$ at a certain average density and disorder realization, repeating projective measurements in the same disorder realization is necessary. Such experiments with ultracold atomic gases in optical lattices should be capable of reaching much larger system sizes than exact diagonalization or the shift-and-invert method, which could give better access to the transition.

In principle, there are also other states that can localize particles such as Mott insulators [82]. In our case, we work at filling 0.5 , where a Mott insulator would not be realized in the BHM in the absence of a dimerization mechanism. Moreover, one is generally interested in physics sufficiently high above the ground state in the context of MBL while the Mott insulator is, strictly speaking, a ground-state phenomenon. In the Mott insulator at, e.g., unit filling, the distribution of densities is $P\left(n_{i}\right) \propto \delta\left(n_{i}-1\right)$, which is clearly different from the behavior in the putative MBL phase [see Fig. 13(c)]. Obviously, the full characterization of a disordered system should rely on a set of experimental measures, including, e.g., decay of inhomogeneous density profiles $[37,42]$ or density distributions as suggested here.

\section{CONCLUSIONS}

We showed that the one-particle density matrix, natural orbitals, and their occupations can be used to reveal the structure of real-space and Fock-space localization in systems of interacting disordered bosons. The real-space localization is observed in the structure of the natural orbitals, in the system-size dependence of the inverse participation ratio, and in the full distribution of densities. The Fock-space localization is uncovered via studying distributions of occupations and densities. Particularly, the distributions of the densities $n_{i}$ and the occupations of natural orbitals $n_{\alpha}$ are smooth functions in the ergodic regime whereas they develop a peak structure in the MBL regime where the peaks are at the possible integer eigenvalues of $\hat{n}_{i}$ and $\hat{n}_{\alpha}$. Based on this observation, we devised a quantitative measure of localization, the average distance to the closest integer of the occupations called occupation distance, and we showed that its system-size dependence is strikingly different in the two phases. This measure can be used to study Fock-space localization for spins, bosons and fermions.

These findings further illustrate the conceptual picture that many-body localization involves localization both in Fock space and in real space. An interesting question pertains to a construction of local conserved charges for the MBL phase of the BHM, i.e., the generalization of l-bits to a system with a large local Hilbert space. The distributions of $n_{i}$ should be accessible in quantum-gas microscope experiments $[37,41,42,44,45]$. It would be interesting to extend our analysis beyond just the expectation values $n_{i}$ to a prediction of projective measurements in the MBL phase.

\section{ACKNOWLEDGMENTS}

We acknowledge useful discussions with V. Alba, J. H. Bardarson, I. Bloch, M. Knap, and F. Pollmann. We thank J. Zakrzewski for pointing out Ref. [53] to us. We are indebted to D. Luitz for sending us data from Ref. [21]. 
[2] R. Nandkishore and D. A. Huse, Many-Body Localization and Thermalization in Quantum Statistical Mechanics, Ann. Rev. Cond. Matt. Phys. 6, 15 (2015).

[3] E. Altman, Many-body localization and quantum thermalization, Nat. Phys. 14, 979 (2018).

[4] F. Alet and N. Laflorencie, Many-body localization: An introduction and selected topics, C. R. Phys. 19, 498 (2018).

[5] D. A. Abanin, E. Altman, I. Bloch, and M. Serbyn, Colloquium: Many-body localization, thermalization, and entanglement, Rev. Mod. Phys. 91, 021001 (2019).

[6] P. W. Anderson, Absence of Diffusion in Certain Random Lattices, Phys. Rev. 109, 1492 (1958).

[7] I. V. Gornyi, A. D. Mirlin, and D. G. Polyakov, Interacting Electrons in Disordered Wires: Anderson Localization and Low-T Transport, Phys. Rev. Lett. 95, 206603 (2005).

[8] D. Basko, I. Aleiner, and B. Altshuler, Metal-insulator transition in a weakly interacting many-electron system with localized single-particle states, Ann. Phys. (N.Y.) 321, 1126 (2006).

[9] M. Serbyn, Z. Papić, and D. A. Abanin, Universal Slow Growth of Entanglement in Interacting Strongly Disordered Systems, Phys. Rev. Lett. 110, 260601 (2013).

[10] D. A. Huse, R. Nandkishore, and V. Oganesyan, Phenomenology of fully many-body-localized systems, Phys. Rev. B 90, 174202 (2014).

[11] J. Z. Imbrie, V. Ros, and A. Scardicchio, Local integrals of motion in many-body localized systems, Ann. Phys. (Leipzig) 529, 1600278.

[12] J. Z. Imbrie, Diagonalization and Many-Body Localization for a Disordered Quantum Spin Chain, Phys. Rev. Lett. 117, 027201 (2016).

[13] B. Bauer and C. Nayak, Area laws in a many-body localized state and its implications for topological order, J. Stat. Mech. Theor. Exp. 2013, P09005 (2013).

[14] J. A. Kjäll, J. H. Bardarson, and F. Pollmann, ManyBody Localization in a Disordered Quantum Ising Chain, Phys. Rev. Lett. 113, 107204 (2014).

[15] M. Friesdorf, A. H. Werner, W. Brown, V. B. Scholz, and J. Eisert, Many-Body Localization Implies that Eigenvectors are Matrix-Product States, Phys. Rev. Lett. 114, 170505 (2015).

[16] J. H. Bardarson, F. Pollmann, and J. E. Moore, Unbounded Growth of Entanglement in Models of ManyBody Localization, Phys. Rev. Lett. 109, 017202 (2012).

[17] M. Žnidarič, T. Prosen, and P. Prelovšek, Many-body localization in the Heisenberg $X X Z$ magnet in a random field, Phys. Rev. B 77, 064426 (2008).

[18] L. F. Santos, Integrability of a disordered Heisenberg spin-1/2 chain, J. Phys. A 37, 4723 (2004).

[19] V. Oganesyan and D. A. Huse, Localization of interacting fermions at high temperature, Phys. Rev. B 75, 155111 (2007).

[20] A. Pal and D. A. Huse, Many-body localization phase transition, Phys. Rev. B 82, 174411 (2010).

[21] D. J. Luitz, N. Laflorencie, and F. Alet, Many-body localization edge in the random-field Heisenberg chain, Phys. Rev. B 91, 081103 (2015).

[22] Y. Bar Lev, G. Cohen, and D. R. Reichman, Absence of Diffusion in an Interacting System of Spinless Fermions on a One-Dimensional Disordered Lattice, Phys. Rev. Lett. 114, 100601 (2015).

[23] S. Bera, H. Schomerus, F. Heidrich-Meisner, and J. H.
Bardarson, Many-Body Localization Characterized from a One-Particle Perspective, Phys. Rev. Lett. 115, 046603 (2015).

[24] S. P. Lim and D. N. Sheng, Many-body localization and transition by density matrix renormalization group and exact diagonalization studies, Phys. Rev. B 94, 045111 (2016).

[25] V. Khemani, F. Pollmann, and S. L. Sondhi, Obtaining Highly Excited Eigenstates of Many-Body Localized Hamiltonians by the Density Matrix Renormalization Group Approach, Phys. Rev. Lett. 116, 247204 (2016).

[26] V. Khemani, D. N. Sheng, and D. A. Huse, Two Universality Classes for the Many-Body Localization Transition, Phys. Rev. Lett. 119, 075702 (2017).

[27] J. Šuntajs, J. Bonča, T. Prosen, and L. Vidmar, Quantum chaos challenges many-body localization, arXiv:1905.06345.

[28] P. Sierant, D. Delande, and J. Zakrzewski, Thouless Time Analysis of Anderson and Many-Body Localization Transitions, Phys. Rev. Lett. 124, 186601 (2020).

[29] D. A. Abanin, J. H. Bardarson, G. De Tomasi, S. Gopalakrishnan, V. Khemani, S. A. Parameswaran, F. Pollmann, A. C. Potter, M. Serbyn, and R. Vasseur, Distinguishing localization from chaos: challenges in finite-size systems, arXiv:1911.04501.

[30] R. K. Panda, A. Scardicchio, M. Schulz, S. R. Taylor, and M. Žnidarič, Can we study the many-body localisation transition?, EPL 128, 67003 (2019).

[31] N. Macé, F. Alet, and N. Laflorencie, Multifractal Scalings Across the Many-Body Localization Transition, Phys. Rev. Lett. 123, 180601 (2019).

[32] A. Goremykina, R. Vasseur, and M. Serbyn, Analytically Solvable Renormalization Group for the ManyBody Localization Transition, Phys. Rev. Lett. 122, 040601 (2019).

[33] P. T. Dumitrescu, A. Goremykina, S. A. Parameswaran, M. Serbyn, and R. Vasseur, Kosterlitz-Thouless scaling at many-body localization phase transitions, Phys. Rev. B 99, 094205 (2019).

[34] A. Morningstar and D. A. Huse, Renormalization-group study of the many-body localization transition in one dimension, Phys. Rev. B 99, 224205 (2019).

[35] J. Šuntajs and J. Bonča and T. Prosen and L. Vidmar, Ergodicity Breaking Transition in Finite Disordered Spin Chains, arXiv:2004.01719.

[36] N. Laflorencie, G. Lemari, and N. Macé, Chain breaking and Kosterlitz-Thouless scaling at the many-body localization transition, arXiv:2004.02861.

[37] M. Schreiber, S. S. Hodgman, P. Bordia, H. P. Lüschen, M. H. Fischer, R. Vosk, E. Altman, U. Schneider, and I. Bloch, Observation of many-body localization of interacting fermions in a quasirandom optical lattice, Science 349, 842 (2015).

[38] J. Smith, A. Lee, P. Richerme, B. Neyenhuis, P. W. Hess, P. Hauke, M. Heyl, D. A. Huse, and C. Monroe, Many-body localization in a quantum simulator with programmable random disorder, Nat. Phys. 12, 907 (2016).

[39] K. Xu, J.-J. Chen, Y. Zeng, Y.-R. Zhang, C. Song, W. Liu, Q. Guo, P. Zhang, D. Xu, H. Deng, K. Huang, H. Wang, X. Zhu, D. Zheng, and H. Fan, Emulating Many-Body Localization with a Superconducting Quantum Processor, Phys. Rev. Lett. 120, 050507 (2018).

[40] P. Roushan, C. Neill, J. Tangpanitanon, V. M. Bastidas, A. Megrant, R. Barends, Y. Chen, Z. Chen, B. Chiaro, 
A. Dunsworth, A. Fowler, B. Foxen, M. Giustina, E. Jeffrey, J. Kelly, E. Lucero, J. Mutus, M. Neeley, C. Quintana, D. Sank, A. Vainsencher, J. Wenner, T. White, H. Neven, D. G. Angelakis, and J. Martinis, Spectroscopic signatures of localization with interacting photons in superconducting qubits, Science 358, 1175 (2017).

[41] T. Kohlert, S. Scherg, X. Li, H. P. Lüschen, S. Das Sarma, I. Bloch, and M. Aidelsburger, Observation of Many-Body Localization in a One-Dimensional System with a Single-Particle Mobility Edge, Phys. Rev. Lett. 122, 170403 (2019).

[42] J.-Y. Choi, S. Hild, J. Zeiher, P. Schauß, A. RubioAbadal, T. Yefsah, V. Khemani, D. A. Huse, I. Bloch, and C. Gross, Exploring the many-body localization transition in two dimensions, Science 352, 1547 (2016).

[43] A. Rubio-Abadal, J.-Y. Choi, J. Zeiher, S. Hollerith, J. Rui, I. Bloch, and C. Gross, Many-body delocalization in the presence of a quantum bath, Phys. Rev. X 9, 041014 (2019).

[44] A. Lukin, M. Rispoli, R. Schittko, M. E. Tai, A. M. Kaufman, S. Choi, V. Khemani, J. Léonard, and M. Greiner, Probing entanglement in a many-body-localized system, Science 364, 256 (2019).

[45] M. Rispoli, A. Lukin, R. Schittko, S. Kim, M. E. Tai, J. Léonard, and M. Greiner, Quantum critical behaviour at the many-body localization transition, Nature 573, 385 (2019).

[46] B. Chiaro, C. Neill, A. Bohrdt, M. Filippone, F. Arute, K. Arya, R. Babbush, D. Bacon, J. Bardin, R. Barends, S. Boixo, D. Buell, B. Burkett, Y. Chen, Z. Chen, R. Collins, A. Dunsworth, E. Farhi, A. Fowler, B. Foxen, C. Gidney, M. Giustina, M. Harrigan, T. Huang, S. Isakov, E. Jeffrey, Z. Jiang, D. Kafri, K. Kechedzhi, J. Kelly, P. Klimov, A. Korotkov, F. Kostritsa, D. Landhuis, E. Lucero, J. McClean, X. Mi, A. Megrant, M. Mohseni, J. Mutus, M. McEwen, O. Naaman, M. Neeley, M. Niu, A. Petukhov, C. Quintana, N. Rubin, D. Sank, K. Satzinger, A. Vainsencher, T. White, Z. Yao, P. Yeh, A. Zalcman, V. Smelyanskiy, H. Neven, S. Gopalakrishnan, D. Abanin, M. Knap, J. Martinis, and P. Roushan, Growth and preservation of entanglement in a many-body localized system, arXiv:1910.06024.

[47] P. Sierant and J. Zakrzewski, Many-body localization of bosons in optical lattices, New J. Phys. 20, 043032 (2018).

[48] P. Sierant and J. Zakrzewski, Model of level statistics for disordered interacting quantum many-body systems, Phys. Rev. B 101, 104201 (2020).

[49] T. B. Wahl, A. Pal, and S. H. Simon, Signatures of the many-body localized regime in two dimensions, Nat. Phys. 15, 164 (2019).

[50] T. Orell, A. A. Michailidis, M. Serbyn, and M. Silveri, Probing the many-body localization phase transition with superconducting circuits, Phys. Rev. B 100, 134504 (2019).

[51] A. Geissler and G. Pupillo, Many-body localization in the two dimensional Bose-Hubbard model, arXiv:1909.09247.

[52] R. Yao and J. Zakrzewski, Many-body localization in Bose-Hubbard model: evidence for the mobility edge, arXiv:2002.00381.

[53] P. Sierant, D. Delande, and J. Zakrzewski, Many-body localization due to random interactions, Phys. Rev. A 95, 021601 (2017).
[54] V. P. Michal, I. L. Aleiner, B. L. Altshuler, and G. V. Shlyapnikov, Finite-temperature fluid-insulator transition of strongly interacting 1D disordered bosons, Proc. Natl. Acad. Sci. 113, E4455 (2016).

[55] G. Bertoli, V. P. Michal, B. L. Altshuler, and G. V. Shlyapnikov, Finite-Temperature Disordered Bosons in Two Dimensions, Phys. Rev. Lett. 121, 030403 (2018).

[56] G. Bertoli, B. L. Altshuler, and G. V. Shlyapnikov, Many-body localization in continuum systems: Twodimensional bosons, Phys. Rev. A 100, 013628 (2019).

[57] W. De Roeck and F. Huveneers, Scenario for delocalization in translation-invariant systems, Phys. Rev. B 90, 165137 (2014).

[58] A. Bols and W. De Roeck, Asymptotic localization in the Bose-Hubbard model, J. Math. Phys. 59, 021901 (2018).

[59] S. Bera, T. Martynec, H. Schomerus, F. HeidrichMeisner, and J. H. Bardarson, One-particle density matrix characterization of many-body localization, Ann. Phys. (Leipzig) 529, 1600356 (2017).

[60] T. L. M. Lezama, S. Bera, H. Schomerus, F. HeidrichMeisner, and J. H. Bardarson, One-particle density matrix occupation spectrum of many-body localized states after a global quench, Phys. Rev. B 96, 060202 (2017).

[61] S.-H. Lin, B. Sbierski, F. Dorfner, C. Karrasch, and F. Heidrich-Meisner, Many-body localization of spinless fermions with attractive interactions in one dimension, SciPost Phys. 4, 002 (2018).

[62] W. Buijsman, V. Gritsev, and V. Cheianov, Many-body localization in the Fock space of natural orbitals, SciPost Phys. 4, 38 (2018).

[63] B. Villalonga, X. Yu, D. J. Luitz, and B. K. Clark, Exploring one-particle orbitals in large many-body localized systems, Phys. Rev. B 97, 104406 (2018).

[64] C. P. Chen, M. Szyniszewski, and H. Schomerus, Manybody localization of zero modes, Phys. Rev. Research 2, 023118 (2020).

[65] S. Roy, J. T. Chalker, and D. E. Logan, Percolation in Fock space as a proxy for many-body localization, Phys. Rev. B 99, 104206 (2019).

[66] D. E. Logan and S. Welsh, Many-body localization in Fock space: A local perspective, Phys. Rev. B 99, 045131 (2019).

[67] S. Inglis and L. Pollet, Accessing Many-Body Localized States through the Generalized Gibbs Ensemble, Phys. Rev. Lett. 117, 120402 (2016).

[68] A. Kshetrimayum, M. Rizzi, J. Eisert, and R. Orús, Tensor Network Annealing Algorithm for Two-Dimensional Thermal States, Phys. Rev. Lett. 122, 070502 (2019).

[69] M. Rigol, Breakdown of Thermalization in Finite OneDimensional Systems, Phys. Rev. Lett. 103, 100403 (2009).

[70] F. Pietracaprina, N. Macé, D. J. Luitz, and F. Alet, Shiftinvert diagonalization of large many-body localizing spin chains, SciPost Phys. 5, 45 (2018).

[71] T. Devakul and R. R. P. Singh, Early Breakdown of Area-Law Entanglement at the Many-Body Delocalization Transition, Phys. Rev. Lett. 115, 187201 (2015).

[72] E. V. H. Doggen, F. Schindler, K. S. Tikhonov, A. D. Mirlin, T. Neupert, D. G. Polyakov, and I. V. Gornyi, Many-body localization and delocalization in large quantum chains, Phys. Rev. B 98, 174202 (2018).

[73] T. Chanda, P. Sierant, and J. Zakrzewski, Time dynamics with matrix product states: Many-body localization transition of large systems revisited, Phys. Rev. B 101, 
035148 (2020).

[74] A. B. Harris, Effect of random defects on the critical behaviour of ising models, Journal of Physics C: Solid State Physics 7, 1671 (1974).

[75] A. Chandran, C. R. Laumann, and V. Oganesyan, Finite size scaling bounds on many-body localized phase transitions, arXiv:1509.04285.

[76] D. J. Luitz, Long tail distributions near the many-body localization transition, Phys. Rev. B 93, 134201 (2016).

[77] X. Yu, D. J. Luitz, and B. K. Clark, Bimodal entanglement entropy distribution in the many-body localization transition, Phys. Rev. B 94, 184202 (2016).

[78] D. M. Kennes and C. Karrasch, Entanglement scaling of excited states in large one-dimensional many-body local- ized systems, Phys. Rev. B 93, 245129 (2016).

[79] J. M. Zhang and R. X. Dong, Exact diagonalization: the Bose-Hubbard model as an example, Eur. J. Phys. 31, $591(2010)$

[80] D. Raventós, T. Graß, M. Lewenstein, and B. Juliá-Díaz, Cold bosons in optical lattices: a tutorial for exact diagonalization, J. Phys. B 50, 113001 (2017).

[81] D. N. Page, Average Entropy of a Subsystem, Phys. Rev. Lett. 71, 1291 (1993).

[82] I. Bloch, J. Dalibard, and W. Zwerger, Many-body physics with ultracold gases, Rev. Mod. Phys. 80, 885 (2008). 